\title{
Bottom-up fabrication of zwitterionic polymer brushes on intraocular lens for improved biocompatibility
}

This article was published in the following Dove Press journal:

International Journal of Nanomedicine

20 December 2016

Number of times this article has been viewed

\author{
Yuemei Han ${ }^{1, *}$ \\ $\mathrm{Xu} X \mathrm{u}^{\mathrm{l}, *}$ \\ Junmei Tangl,* \\ Chenghui Shen ${ }^{2}$ \\ Quankui Lin ${ }^{1,2}$ \\ Hao Chen ${ }^{1,2}$ \\ 'School of Ophthalmology \& \\ Optometry, Eye Hospital, Wenzhou \\ Medical University, ${ }^{2}$ Wenzhou \\ Institute of Biomaterials and \\ Engineering, Chinese Academy of \\ Sciences, Wenzhou, People's Republic \\ of China \\ *These authors contributed equally \\ to this work
}

\begin{abstract}
Intraocular lens (IOL) is an efficient implantable device commonly used for treating cataracts. However, bioadhesion of bacteria or residual lens epithelial cells on the IOL surface after surgery causes postoperative complications, such as endophthalmitis or posterior capsular opacification, and leads to loss of sight again. In the present study, zwitterionic polymer brushes were fabricated on the IOL surface via bottom-up grafting procedure. The attenuated total reflection-Fourier transform infrared and contact angle measurements indicated successful surface modification, as well as excellent hydrophilicity. The coating of hydrophilic zwitterionic polymer effectively decreased the bioadhesion of lens epithelial cells or bacteria. In vivo intraocular implantation results showed good in vivo biocompatibility of zwitterionic IOL and its effectiveness against postoperative complications.
\end{abstract}

Keywords: RAFT, surface modification, endophthalmitis, PCO, in vivo

\section{Introduction}

Cataract accounts for over half of the cases of blindness worldwide. Surgical removal of opaque lens followed by intraocular lens (IOL) implantation is the most common treatment. However, bioadhesion of bacteria or residual lens epithelial cells (LECs) after surgery easily occurs on the IOL surface due to its intrinsic nature. The bioadhesion on IOL surface causes postoperative complications, such as endophthalmitis or posterior capsular opacification (PCO), and leads to loss of sight again. For example, the adhesion of residual LECs onto the IOL surface after surgery may cause PCO, as a series of cellular processes such as migration, proliferation, and transdifferentiation follow. The incidence of PCO is high and varies between $10 \%$ and $50 \%$ within 3-5 years after implantation. ${ }^{1}$ Postoperative infectious endophthalmitis is another disastrous complication of cataract surgery and often causes severe visual impairment and even loss of sight. Adhesion by bacteria, such as the Gram-positive Micrococcus spp., Staphylococcus epidermidis, and Staphylococcus aureus, and subsequent biofilm development on the IOL, has been regarded as the main cause of infectious endophthalmitis. ${ }^{2}$ These microorganisms adhere onto the IOL after implantation, proliferate and colonize subsequently, and then form biofilms on the implanted IOL postoperatively. ${ }^{3}$ This bioadhesion greatly influences the clinical effects after the IOL implantation.

Surface modification of implantable biomaterials provides an effective way to improve their biocompatibilities and has attracted more and more attention in recent years. ${ }^{4-8}$ Surface modification of IOL materials neither requires any manipulation within eyes nor uses possibly harmful compounds during implantation, which is an attractive
School of Ophthalmology \& Optometry, Eye Hospital, Wenzhou Medical University, 270 Xueyuan Xi Road, Wenzhou 325027, People's Republic of China

Tel/fax +865778806 7962

Email lqk9753I@I26.com 
method to reduce the postoperative complication after the IOL implantation. The earliest IOL surface modification was the surface coating with inert materials like carbon, titanium, or Teflon, which can reduce the LEC adhesion to some extent. ${ }^{9,10}$ Investigations in the recent decade indicate that the surface hydrophilic modification provides a much more effective way to reduce the bioadhesion. For example, treating IOL surface with plasma or immobilizing with heparin, poly(ethylene glycol), or phosphorylcholine can reduce the bioadhesion better than coating with inert materials. ${ }^{11-16}$ However, bioadhesion can still be found on coated hydrophilic surfaces, which may be due to the undesired surface coating by common hydrophilic molecules. A more effective antifouling coating is essential for IOL surface modifications.

Surface-initiated living radical polymerization reacobtaining well-defined polymer brushes provide a good alternative for the surface modification of biomedical materials. Recently, poly(carboxybetaine methacrylate), a superhydrophilic zwitterionic polymer, has been used to modify the silicone surface via the atom transfer radical polymerization approach. Excellent antifouling properties and long-term stability of the zwitterionic polymer-modified silicone were observed. ${ }^{17}$ Reversible addition-fragmentation chain-transfer (RAFT) polymerization is another living radical polymerization and has enjoyed widespread acceptance as a modification technique due to its capability to control polymerization of diverse monomers under mild reaction conditions without the requirement of metal catalysts. ${ }^{18,19}$ 2-Methacryloyloxyethyl phosphorylcholine (MPC) is another zwitterionic material, and can provide an artificial cell membrane structure at the surface due to its biomimetic structure and serve as an excellent biointerface between artificial and biological systems. ${ }^{20}$ Herein, surface-initiated RAFT (SI-RAFT) was carried out for bottom-up grafting MPC brushes on IOL surface for anti-bioadhesion applications. The hydrophilicity of the coatings was investigated, and the anti-bioadhesion properties were tested by $S$. aureus adhesion and LEC adhesion assays. The zwitterionic polymer-modified IOL was then implanted into the animal eyes by clinical cataract surgery procedure to evaluate the in vivo biocompatibility, as well as the postoperative complication prevention effect.

\section{Materials and methods \\ Materials}

MPC, 4-cyano-4-(phenyl-carbonothioylthio) pentanoic acid (CPCTTPA), 2-morpholino-ethanesulfonic acid, 4,4'azobis-(4-cyanovaleric acid) (V501), (3-aminopropyl) triethoxysilane (APTES), $N$-(3-dimethylaminopropyl)$N^{\prime}$-ethylcarbodiimide hydrochloride (EDC), $N$-hydroxysulfosuccinimide sodium salt (NHSS), and fluorescein diacetate were purchased from Sigma-Aldrich Co. (St Louis, MO, USA). LIVE/DEAD BacLight bacterial viability kit (L-7012) was supplied by Invitrogen. Masson' Trichrome staining kits were purchased from Leagene Biotech Co., Ltd (Beijing, People's Republic of China). Silicone (polydimethylsiloxane, PDMS), prepared from Sylgard ${ }^{\circledR}$ 184 of Dow Corning (Midland, MI, USA), was used as hydrophobic IOL material substrate for characterization. ${ }^{21}$ Foldable IOL for animal experiments was supplied by Alcon (Fort Worth, TX, USA; AcrySof ${ }^{\circledR}$ SN60WF; diameter of optic: $6 \mathrm{~mm}$ ). Human LEC line (HLE B3, CRL-11421 ${ }^{\mathrm{TM}}$ ) was originally obtained from American Type Culture Collection (ATCC) (Manassas, VA, USA). The clinical postoperative administration drugs were obtained from Eye Hospital of Wenzhou Medical University.

\section{Surface modification of the IOL and characterization}

The PDMS silicone materials or IOL was used as the substrate for zwitterionic surface modification. The fabrication procedure was similar to that reported in the previous publication. ${ }^{22}$ As illustrated in Figure 1, each experiment was initiated by aminolysis by treatment with $2 \%$ (v/v) APTES, followed by coupling with carboxyl group-containing RAFT agent (CPCTTPA) by EDC/NHSS chemistry. Then, the samples were put in glass vials with $1 \mathrm{mg} / \mathrm{mL} \mathrm{V501} \mathrm{and}$ $100 \mathrm{mg} / \mathrm{mL}$ MPC in 2-morpholino-ethanesulfonic acid buffer ( $\mathrm{pH}$ 5.2). After sealing and degassing, the reaction was carried out in a microwave reactor (Initiator 60; Biotage AB, Uppsala, Sweden) at $60^{\circ} \mathrm{C}$ for 6 hours. After reaction, the poly(MPC) (PMPC)-modified IOL materials were washed with pure water and dried for further use. The chemical composition of the surface-modified samples was determined by X-ray photoelectron spectroscopy (XPS) (PerkinElmer Inc., Waltham, MA, USA). Hydrophilicity of the coating was measured by water contact angle (WCA) analysis (OCA20; DataPhysics Instruments GmbH, Filderstadt, Germany).

\section{In vitro LEC adhesion test}

LEC line derived from human lens was cultured and used to evaluate the initial cell adhesion or proliferation on the IOL materials. ${ }^{21}$ After confluence, the cells were digested by $0.25 \%$ trypsin. The detached cells were collected and resuspended in Rosewell Park Memorial Institute 


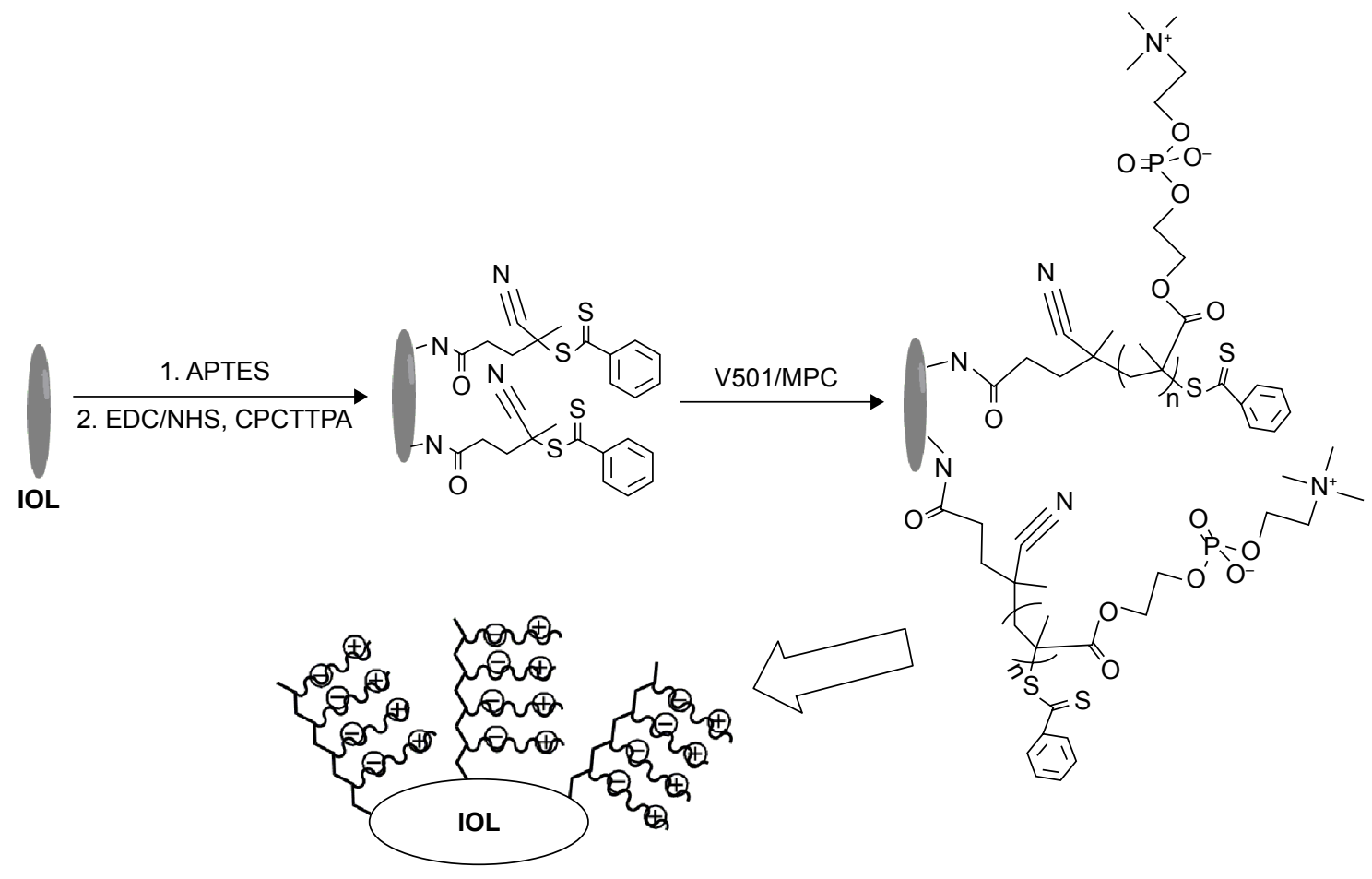

Figure I Schematic illustration of surface-initiated RAFT polymerization of MPC on IOL surface.

Abbreviations: RAFT, reversible addition-fragmentation chain-transfer; MPC, 2-methacryloyloxyethyl phosphorylcholine; IOL, intraocular lens; APTES, (3-aminopropyl) triethoxysilane; EDC, N-(3-dimethylaminopropyl)- $N^{\prime}$-ethylcarbodiimide hydrochloride; NHS, N-hydroxysulfosuccinimide sodium; CPCTTPA, 4-cyano-4-(phenylcarbonothioylthio) pentanoic acid; V50I, 2-morpholino-ethanesulfonic acid, 4,4'-azobis-(4-cyanovaleric acid).

1640 medium. Then, the cells were distributed onto 24-well plates containing the PMPC-grafted IOL materials at a seeding density of $5.0 \times 10^{3}$ cells per well. After 24 - or 72-hour incubation, the cells were stained by fluorescein diacetate and visualized by a fluorescence microscope (Nikon Corporation, Tokyo, Japan). Fluorescent images were acquired at $\times 20$ objective under fluorescein filter (488 nm/excitation). The initial cell adhesion and distribution were measured. The cell density on the substrates was calculated as well.

\section{In vitro bacterial adsorption test}

A Gram-positive bacterial species, $S$. aureus, was used in this work. The bacteria were cultured overnight on LuriaBertani agar plates at $37^{\circ} \mathrm{C}$. Several colonies were inoculated in Luria-Bertani broth. After 16-hour incubation under gentle shaking, the bacteria were collected by centrifugation at $4,000 \mathrm{rpm}$ for 5 minutes and resuspended in sterile phosphatebuffered saline (PBS) at a density of $1 \times 10^{5}$ cells $/ \mathrm{mL}$. The substrates were first immersed in PBS for 30 minutes for equilibrium and then transferred to a 24-well plate which contained $1 \mathrm{~mL}$ of bacterial solution in each well. After incubation for 4 hours under $37^{\circ} \mathrm{C}$ and $5 \% \mathrm{CO}_{2}$, the substrates were washed three times with sterile PBS under gentle shaking and stained with LIVE/DEAD BacLight. The bacterial adhesion was determined by a fluorescence microscope.

\section{In vivo intraocular implantation and evaluation}

This study was approved by the Laboratory Animal Ethics Committee of Wenzhou Medical University. The rabbits were treated in accordance with guidelines set forth by the Association for Research in Vision and Ophthalmology. In vivo intraocular implantation and evaluation were carried out according to our previous publications. ${ }^{21}$ In detail, the in vivo biocompatibility of zwitterionic IOLs was evaluated using 2-month old New Zealand White rabbits. Phacoemulsification followed by lens suction was carried out on the left eye of each rabbit under general anesthesia with the assistance of phacoemulsification instrument (Alcon). Postoperative topical therapy included combination Levofloxacin eye drops and Tobramycin-dexamethasone ointment during the first postoperative week and prednisolone acetate drops, which were tapered during the first and second postoperative weeks.

The eyes were dilated and evaluated by slit lamp microscopy observation postoperatively. The animals were anesthetized and executed humanely with air embolism. The ocular tissue slices were obtained according to the standard 
procedure, stained with hematoxylin-eosin, and observed under microscope. ${ }^{21}$

\section{Results and discussion}

\section{Surface modification of the IOL and characterization}

The bottom-up fabrication of PMPC brushes on the IOL surface is schematically shown in Figure 1. Primary amine groups were generated on IOL material surfaces by silanization via APTES hydrolysis, followed by RAFT agent covalent binding via coupling $-\mathrm{COOH}$ on RAFT agent with $-\mathrm{NH}_{2}$ on the surface by EDC/NHSS chemistry. The RAFT agent-modified surfaces were used for the surfaceinitiated RAFT polymerization of MPC, generating PMPC brush on surface. XPS was used to analyze the changes in elemental composition of the surface in each step. As shown in Figure 2A, pristine PDMS silicone surface consisted mainly of carbon, oxygen, and silicon elements. Four XPS peaks with binding energies at 530.9, 283.9, 149.9, and $98.9 \mathrm{eV}$ were discernible in the wide-scan spectrum of the bare silicone IOL material surface, which were attributed to $\mathrm{O}_{1 \mathrm{~s}}, \mathrm{C}_{1 \mathrm{~s}}, \mathrm{Si}_{2 \mathrm{~s}}$, and $\mathrm{Si}_{2 \mathrm{p}}$ signals, respectively. After aminolysis or RAFT agent coupling, minor peaks at 397.8 or $399.8 \mathrm{eV}$ appeared on the spectra, which were due to the $\mathrm{N}_{1 \mathrm{~s}}$ signal on the surface, as indicated by the magnified view of the spectra (Figure 2B). In addition, there was a minor $\mathrm{S}_{1 \mathrm{~s}}$ signal peak after the RAFT agent immobilization, which was due to the carbonothioylthio group in CPCTTPA (Figure 2C). There were obvious peaks of $\mathrm{N}_{1 \mathrm{~s}}$ and $\mathrm{P}_{2 \mathrm{p}}$ appearing after the surfaceinitiated RAFT polymerization of MPC, which came from the $\mathrm{N}$ and $\mathrm{P}$ elements in MPC molecules, as well as the $\mathrm{N}$ element of the APTES and the RAFT agent (Figure 2B and C). It can also be seen from Figure $2 \mathrm{C}$ that the signal intensity of the $\mathrm{Si}_{2 \mathrm{~s}}$ and $\mathrm{Si}_{2 \mathrm{p}}$ was reduced in the PMPC-modified

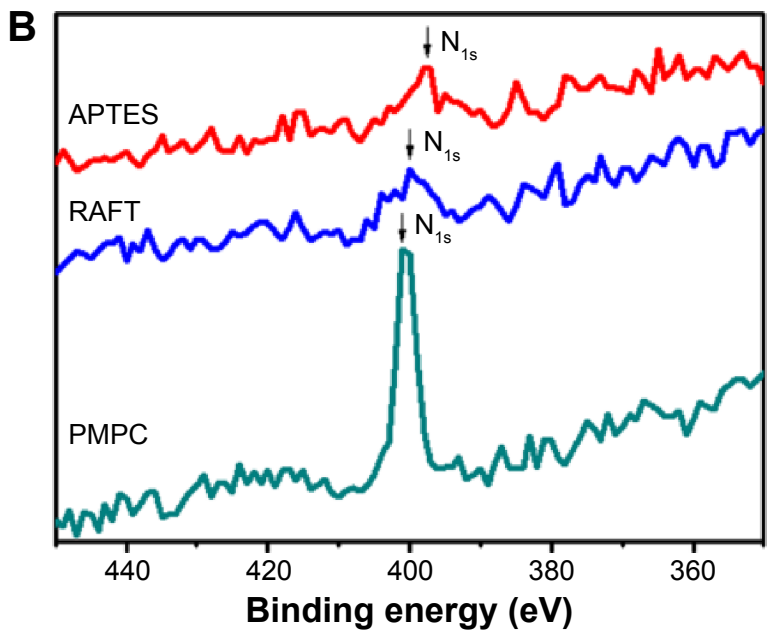

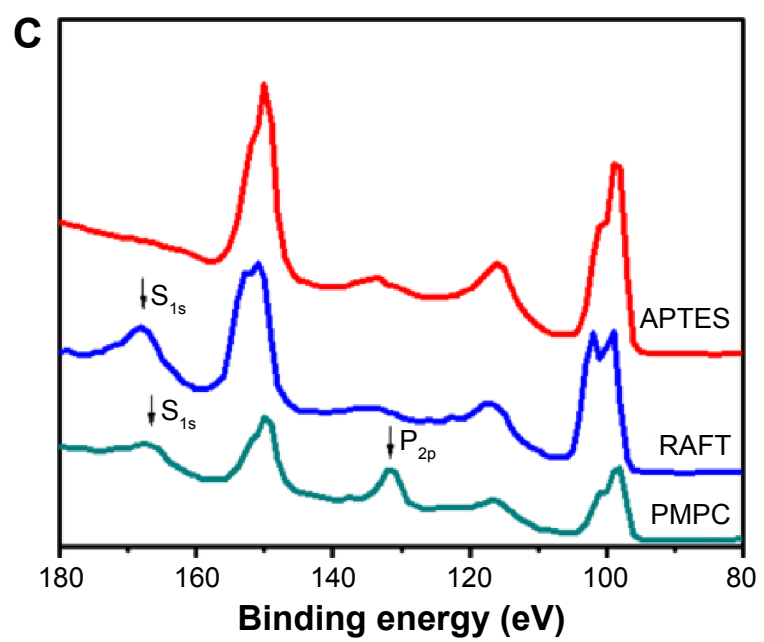

Figure 2 XPS pattern of silicone IOL materials and materials after APTES aminolysis or RAFT agent immobilization, followed by SI-RAFT polymerization of MPC. Notes: (A) The full XPS pattern. (B) The amplified view of the pattern in 350-450 eV. (C) The amplified view of the pattern in $80-180 \mathrm{eV}$.

Abbreviations: XPS, X-ray photoelectron spectroscopy; IOL, intraocular lens; APTES, (3-aminopropyl) triethoxysilane; RAFT, reversible addition-fragmentation chaintransfer; SI-RAFT, surface initiated RAFT; MPC, 2-methacryloyloxyethyl phosphorylcholine; PMPC, poly(MPC). 


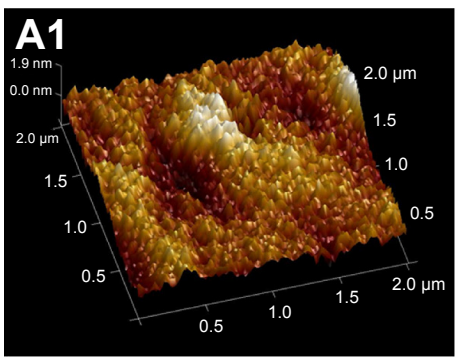

A2
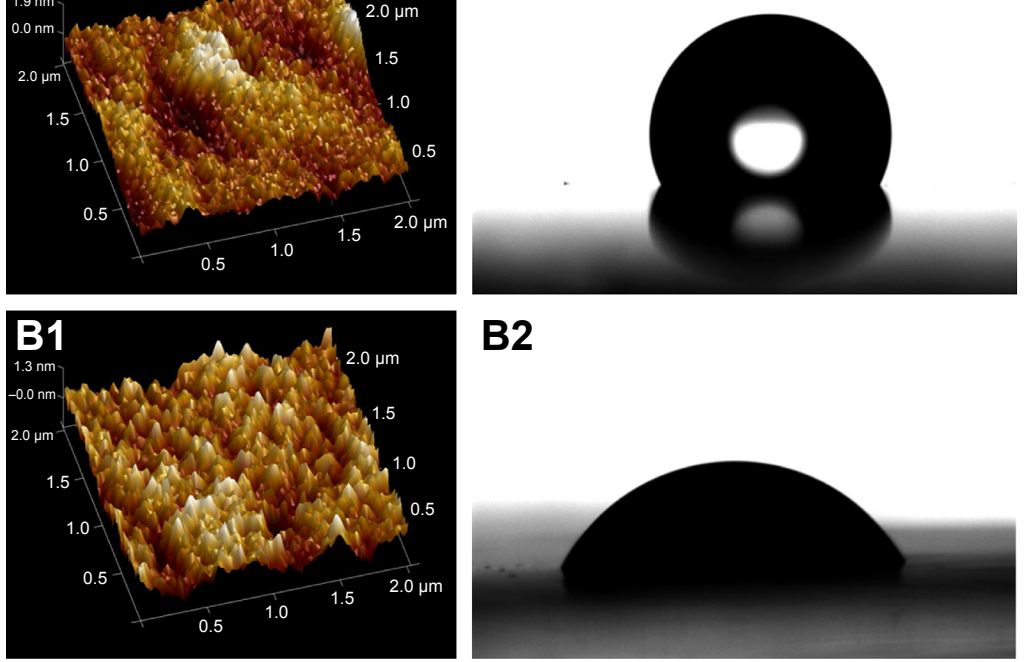

B2

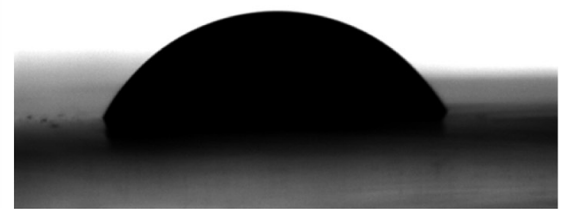

A3

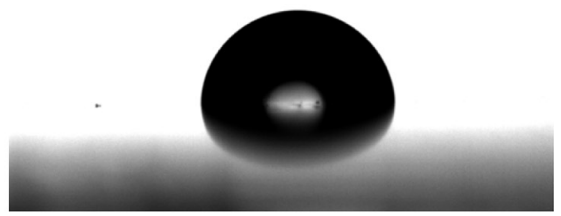

B3

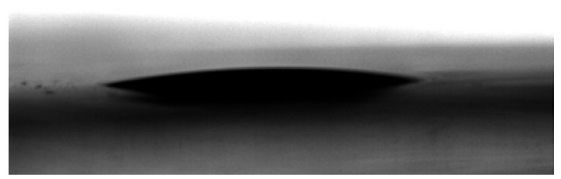

Figure 3 The AFM images (AI and B I) and water contact angle of silicone IOL materials before (AI-A3) and after (B I-B3) PMPC modification. The contact angle photos were taken immediately after the water drop contacted materials (A2 and B2) and after 480 seconds (A3 and B3).

Abbreviations: AFM, atomic force microscopy; IOL, intraocular lens; PMPC, poly(2-methacryloyloxyethyl phosphorylcholine).

surface. As the XPS detection thickness was $<10 \mathrm{~nm}$, the coating of PMPC on the surface decreased the substrate element signals. ${ }^{23}$ These results clearly indicated that the MPC moieties can be introduced onto the silicone IOL surface by SI-RAFT polymerization.

Atomic force microscopy was used to characterize the surface properties of the IOL materials before and after PMPC coating. As shown in Figure 3, no matter with or without PMPC coating, the IOL materials were very homogeneous and smooth. The vertical distances of the main peaks and valleys were 1.41 and $2.72 \mathrm{~nm}$ on the unmodified and PMPC-coated surfaces. The Ra calculated in roughness analysis was 0.25 and $3.84 \mathrm{~nm}$, respectively. These results indicated that although the PMPC modification slightly increased the roughness, the material surface was homogeneous and smooth. WCA was used to characterize the surface wettability of the IOL material before and after PMPC brush modification (Figure 3). The bare silicone IOL materials were moderately hydrophobic, with a WCA of $114.2^{\circ}$ (Figure 3A2). The zwitterionic PMPC coating improved the surface hydrophilicity effectively. The instantaneous WCA on PMPC-modified surface was $\sim 34^{\circ}$ (Figure 3B2), and continued to decrease and reached $<5^{\circ}$ after equilibrium for 6 minutes (Figure 3B3), whereas the WCA on bare substrate surface remained at $113.4^{\circ}$ (Figure 3A3). The decrease of the WCA not only evidently proved the PMPC coating via SI-RAFT but also showed the excellent hydrophilicity of the modified surface.

\section{In vitro anti-bioadhesion evaluation}

The reduction of bioadhesion plays an important role in the IOL material biocompatibility after implantation. The residual LECs after surgery may adhere to the IOL material surface and proliferate between the surface and capsule, which is the leading cause of secondary cataract. In this study, the PMPC coating was generated on the IOL material surface via SI-RAFT to reduce the bioadhesion. The in vitro LEC adhesion was investigated. As shown in Figure 4, the initial

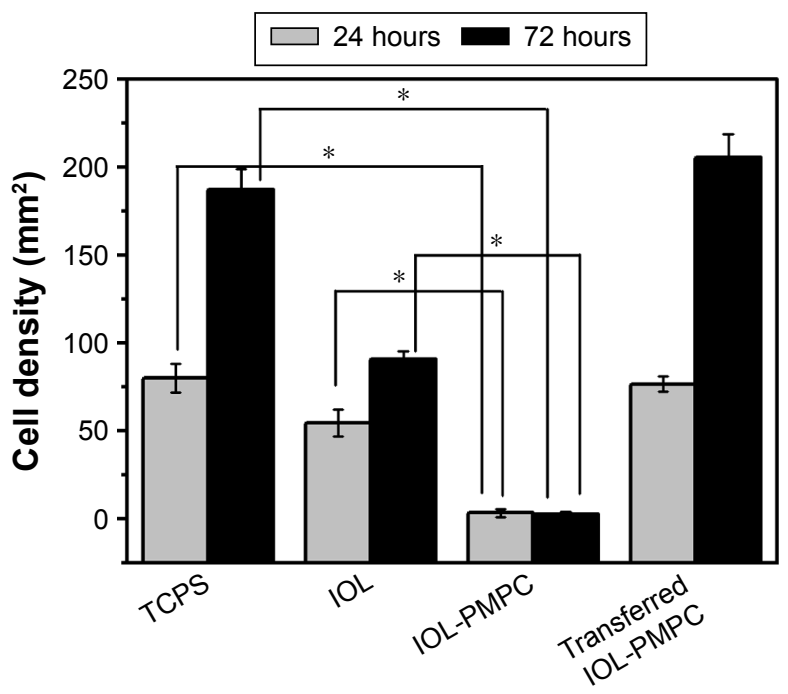

Figure 4 Cell densities on different surfaces after culturing for 24 and 72 hours. Notes: Transferred IOL-PMPC refers to that the cells were originally cultured on IOLPMPC plate for 24 hours and then the culture supernatant was transferred onto TCPS. *Indicates $P<0.05$.

Abbreviations: IOL, intraocular lens; PMPC, poly(2-methacryloyloxyethyl phosphorylcholine); TCPS, tissue culture polystyrene. 

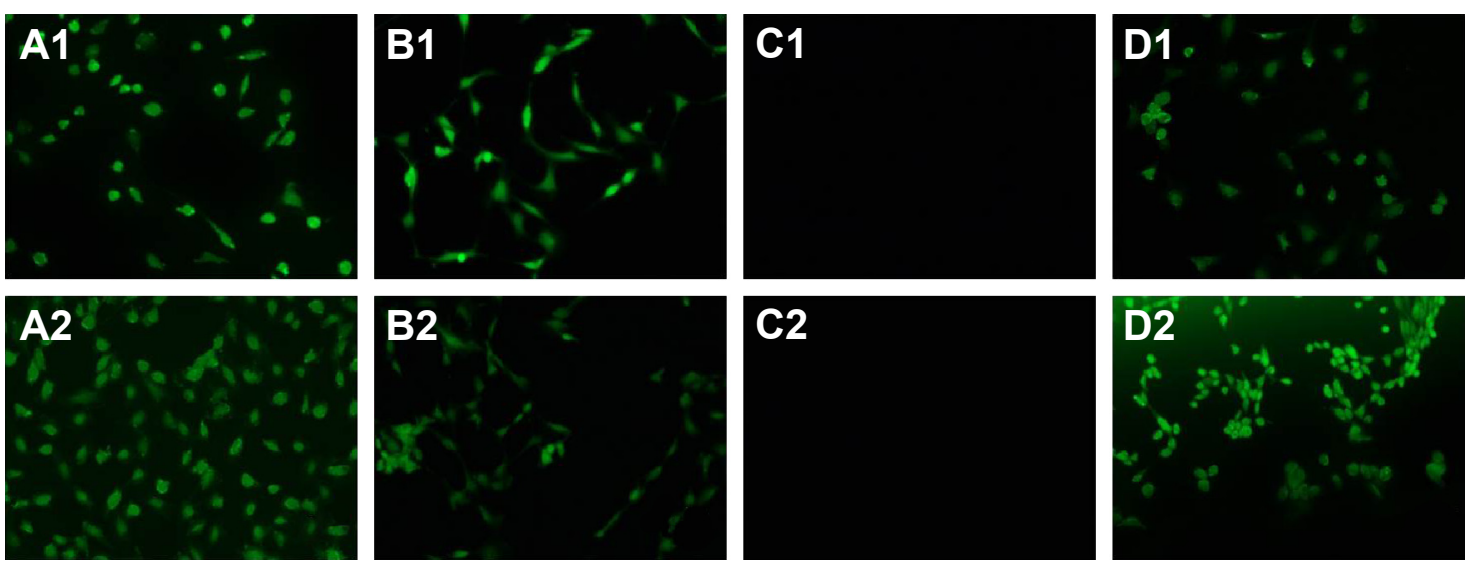

Figure 5 The representative fluorescent images of the LECs cultured on surface of different materials for (A I-DI) 24 and (A2-D2) 72 hours. (AI and A2) TCPS. (B I and B2) Silicone IOL materials. (CI and C2) IOL materials surface coated with PMPC brushes. (DI and D2) Cells were originally cultured on IOL-PMPC plate for 24 hours, and then the culture supernatant was transferred onto TCPS. The magnification is $\times 200$.

Abbreviations: LECs, lens epithelial cells; TCPS, tissue culture polystyrene; IOL, intraocular lens; PMPC, poly(2-methacryloyloxyethyl phosphorylcholine).

LEC adhesion density on tissue culture polystyrene (TCPS) plate was $80.1 \mathrm{cells} / \mathrm{mm}^{2}$, while the density on the bare IOL materials was $\sim 54.5$ cells $/ \mathrm{mm}^{2}$. The introduction of PMPC coating on the surface greatly reduced the LEC adhesion, with almost no cell detected on the coated surface. Meanwhile, when the supernatant from PMPC-coated sample well was transferred into another TCPS well, the cells in the medium attached to the TCPS very well, with an initial cell adhesion density of 76.3 cells $/ \mathrm{mm}^{2}$, which was close to that on the TCPS. The cell proliferation behavior showed similar trend as the cell adhesion. Figure 5 shows the representative fluorescent images of the LECs on different material surfaces after 24- or 72-hour incubation, which demonstrates that the PMPC-modified IOL can effectively reduce the cell adhesion and proliferation. Seldom, LECs can be detected later on the modified surface. Due to its hydrophilicity, zwitterionic MPC has been widely used for biomaterials surface modification. The zwitterionic surface shows great promise for minimizing the bioadhesion. ${ }^{24-27}$ Herein, the excellent LEC-resistant property is also attributed to the hydrophilicity. Previous investigations have demonstrated that the adhesion of residual LECs onto the IOL may be the origin of the PCO..$^{21,28,29}$ As a result, the adhesion inhibition can restrain the formation of multicellular secondary membrane resulting from proliferation, migration, and fibrosis of residual LECs on the posterior capsule and eventually reduce the incidence of PCO. ${ }^{21}$

The bacterial adhesion was also tested in this investigation. The Gram-positive $S$. aureus was used as model bacteria in this study. S. aureus is one of the critical pathogenic bacteria in infectious endophthalmitis after IOL implantation, causing much poor prognosis. ${ }^{30}$ Herein, S. aureus was exposed to pristine or PMPC brush-modified silicone IOL material and stained by LIVE/DEAD BacLight bacterial viability kit. The distribution of the attached viable bacteria on the pristine or PMPC brush-functionalized IOL materials was observed by fluorescence microscopy, as shown in Figure 6 . The images revealed that there was a clear distinction between the adherent bacterial number on the pristine and modified substrates. Numerous distinguishable $S$. aureus with green fluorescence, either individually or in small clusters, were distributed on the unmodified surfaces (Figure 6A), which indicated that most of the bacterial cells were viable with the cell membrane intact. However, seldom bacterial adhesion was found on the PMPC brush-modified surface. The PMPC brush modification can also effectively inhibit the bacterial adhesion on the IOL surface.

\section{In vivo ocular implantation}

To evaluate in vivo biocompatibility, the zwitterionic PMPC brush-modified IOL was implanted in the rabbit eye consequently. The slit lamp images were captured postoperatively on days $1-30$. The ocular slices were
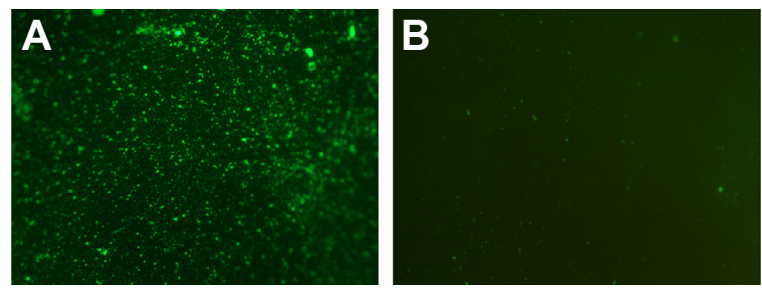

Figure 6 Fluorescence microscopy images of Staphylococcus aureus adhesions on (A) pristine silicone IOL material and (B) PMPC brush-modified silicone IOL material. The magnification is $\times 100$.

Abbreviations: IOL, intraocular lens; PMPC, poly(2-methacryloyloxyethyl phosphorylcholine). 

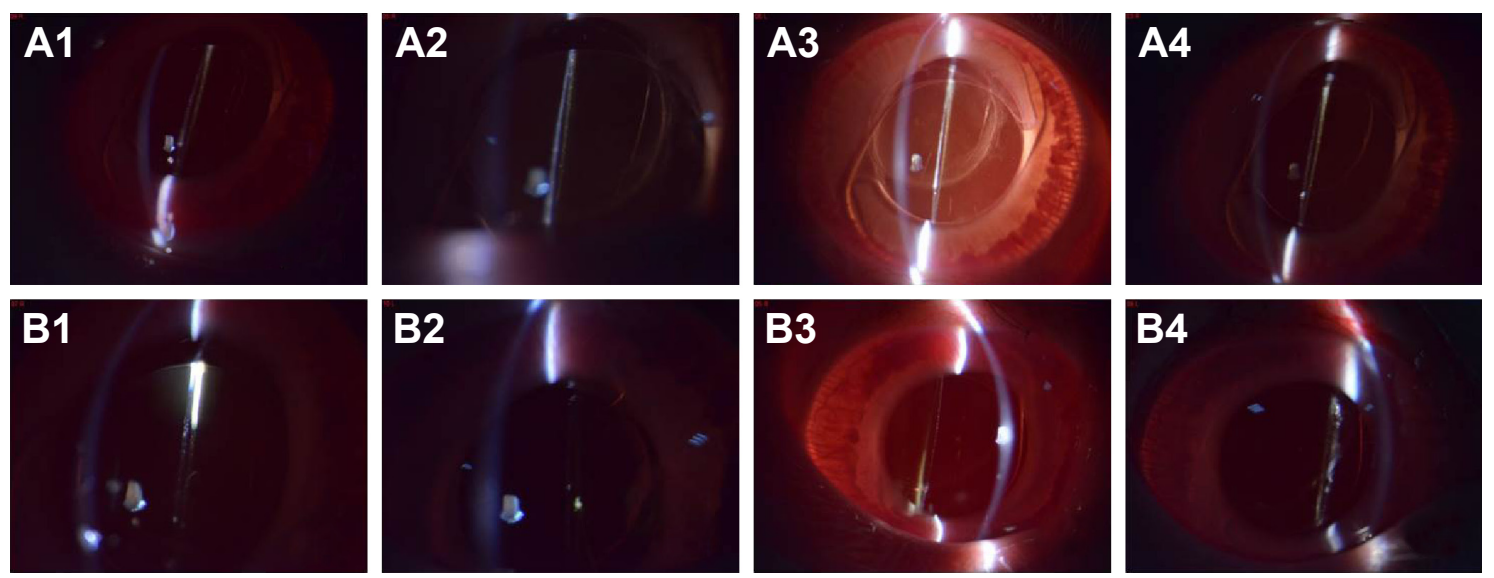

Figure 7 Representative slit lamp images of the (AI-A4) implanted PMPC-modified hydrophobic IOL and (BI-B4) the implanted pristine hydrophobic IOL taken on days $1,3,7$, and 30 . The magnification is $\times 16$.

Abbreviations: PMPC, poly(2-methacryloyloxyethyl phosphorylcholine); IOL, intraocular lens.

examined at day 30 as well. Figure 7 shows the slit lamp images of IOL in rabbit eyes before and after PMPC brush modification. Neither acute anterior chamber inflammation nor case of postoperative infection was found in both groups. The ocular surface and anterior chamber were clear in the observation period, which indicates that there was no acute tissue incompatibility of the IOLs.

The in vivo biocompatibility was then confirmed by histological observation. The ocular tissues were crosscut, stained, and examined. Figure 8 shows the ocular tissue morphology of the PMPC-modified IOL. It can be observed that all the ocular tissues, including cornea, iris, and lens capsule, were visible with their normal morphologies, which indicate the excellent long-term in vivo biocompatibility of the PMPC-modified IOLs. For example, the five-layered corneal structure remained intact, including epithelium, Bowman's membrane, stroma, Descemet membrane, and endothelium. ${ }^{31}$ Iris, a tissue of ocular blood supply, has plenty of blood vessels and chorionic villi. No posterior capsular hyperplasia was found in the lens capsules. The

\section{A}

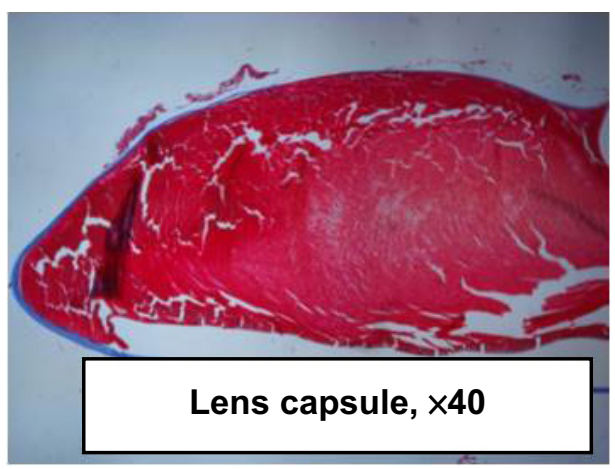

C

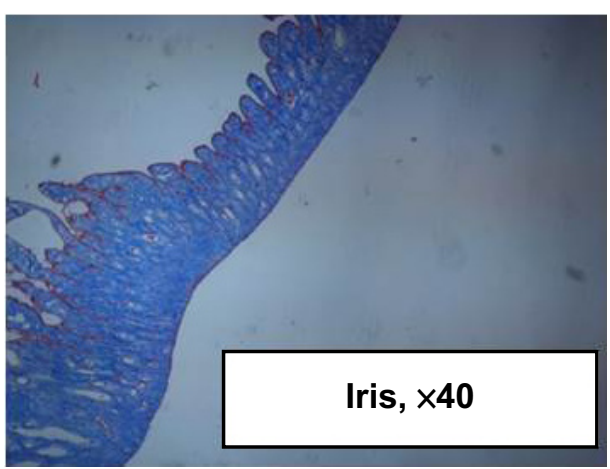

B

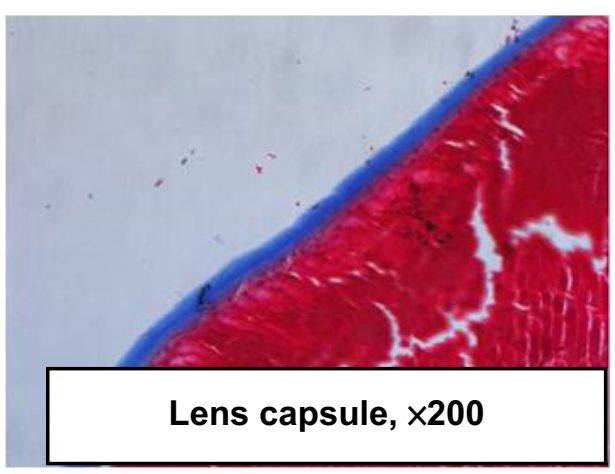

D

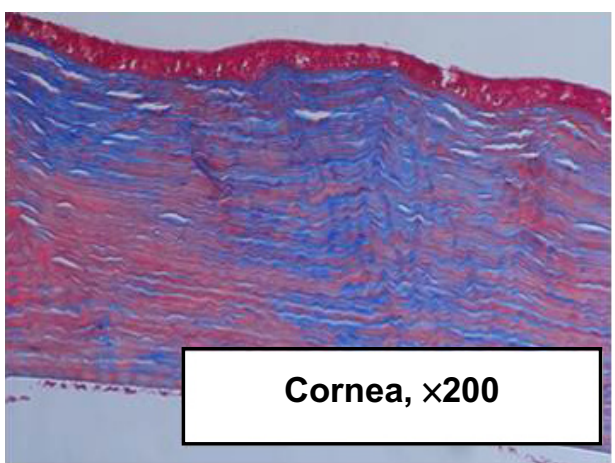

Figure 8 The histochemical stain of the PMPC brushes modified hydrophobic IOL implantation. (A, B) The lens capsule; (C) iris; (D) cornea. Abbreviations: PMPC, poly(2-methacryloyloxyethyl phosphorylcholine); IOL, intraocular lens. 
posterior capsular hyperplasia is the innate formality of PCO. This result was confirmed by the slit lamp observation at postoperative day 30. As shown in Figure 7A4 and $\mathrm{B} 4$, the bare IOL in this case was starting to develop PCO at day 30 (B4). The appearance of severe irregular white patterns on the posterior capsule under the slit light irradiation was attributed to posterior capsular hyperplasia. In contrast, PCO was not found in PMPC brush-modified IOL group during observation. This result reveals that the modification with zwitterionic PMPC can prevent the PCO development.

The bioadhesion mostly occurs on the hydrophobic IOL material surface. The bacterial adhesion on implanted IOL may cause endophthalmitis. The residual LEC adhesion onto the IOL materials after implantation may finally result in PCO. ${ }^{32}$ Various attempts, including improvement in surgical techniques, optimization of IOL design, investigation of novel IOL materials, as well as the use of pharmacological and immunological inhibitors of the LEC proliferation, have been taken to improve the IOL biocompatibility. ${ }^{33-38}$ In addition, surface modification of implantable biomedical devices is an effective way to improve their biocompatibility. ${ }^{3-6,39}$ Cell-resistant surface can be generated via hydrophilic macromolecule immobilization or hydrogel-like surface coating. ${ }^{40-45}$ In this study, we fabricated the zwitterionic PMPC brush onto the hydrophobic IOL surface via SI-RAFT polymerization. Such zwitterionic PMPC brush modification can effectively reduce the initial adhesion of residual LECs or bacteria, and is prospective in reducing the $\mathrm{PCO}$ incidence in vivo. Thus, hydrophilic modification provides an effective way to improve the biocompatibility of the IOL and can effectively prevent the PCO incidence.

\section{Conclusion}

Zwitterionic PMPC brush was introduced onto hydrophobic IOL via surface-initiated RAFT polymerization. The zwitterionic polymer coating greatly improved the surface hydrophilicity of the IOL material. Due to its hydrophilicity, the bioadhesion of LECs and bacteria was distinctly reduced on the PMPC brush-modified surface. Intraocular IOL implantation results revealed an excellent ocular tissue compatibility, as the IOL implantation did not induce pathological changes to the nearby ocular tissues, such as cornea and iris. Compared with the bare IOL, reduced PCO incidence was found on the PMPC brush-modified group. No posterior capsular hyperplasia was found on the lens capsule in the PMPC brush-modified IOLs. These results demonstrate that the hydrophilic modification with zwitterionic PMPC brush can effectively improve the biocompatibility of IOL after implantation.

\section{Acknowledgments}

This work was supported by the Natural Science Foundation of China (31570959, 51203120, 81271703), Natural Science Foundation of Zhejiang Province (LY16H120003, LQ12E03001), Medical \& Health Technology Program of Zhejiang Province (2014KYA149, 2013KYA133), Zhejiang Provincial \& Ministry of Health Research Fund for Medical Science (WKJ-ZJ-1529), S\&T Program of Wenzhou (Y20150083, Y20120201), and Xinmiao Talents Program of Zhejiang Province (2015R413074).

\section{Disclosure}

The authors report no conflicts of interest in this work.

\section{References}

1. Nibourg LM, Gelens E, Kuijer R, Hooymans JMM, van Kooten TG, Koopmans SA. Prevention of posterior capsular opacification. Exp Eye Res. 2015;136:100-115.

2. Saika S, Kawashima Y, Miyamoto T, et al. Pathological findings in lens capsule and silicone intraocular lens extracted from eye with chronic infectious endophthalmitis. JNP J Ophthalmol. 1999;42(6): 456-460.

3. Okajima Y. Biofilm formation by Staphylococcus epidermidis on intraocular lens material. Invest Ophthalmol Vis Sci. 2006;47(7): 2971-2975.

4. Lin QK, Ren KF, Ji J. Hyaluronic acid and chitosan-DNA complex multilayered thin film as surface-mediated nonviral gene delivery system. Colloids Surf B Biointerfaces. 2009;74(1):298-303.

5. Lin QK, Ding X, Qiu FY, Song XX, Fu GS, Ji J. In situ endothelialization of intravascular stents coated with an anti-CD34 antibody functionalized heparin-collagen multilayer. Biomaterials. 2010;31(14):4017-4025.

6. Lin QK, Van JJ, Qiu FY, Song XX, Fu GS, Ji J. Heparin/collagen multilayer as a thromboresistant and endothelial favorable coating for intravascular stent. J Biomed Mater Res A. 2011;96(1):132-141.

7. Wu GS, Li PH, Feng HQ, Zhang XM, Chu PK. Engineering and functionalization of biomaterials via surface modification. J Mater Chem B. 2015;3(10):2024-2042.

8. Chu PK. Surface engineering and modification of biomaterials. Thin Solid Films. 2013;528:93-105.

9. Li DJ, Cui FZ, Gu HQ. F+ ion implantation induced cell attachment on intraocular lens. Biomaterials. 1999;20(20):1889-1896.

10. Werner L, Legeais JM, Nagel MD, Renard G. Neutral red assay of the cytotoxicity of fluorocarbon-coated polymethylmethacrylate intraocular lenses in vitro. J Biomed Mater Res. 1999;48(6):814-819.

11. Huang XD, Li HY, Lin L, Yao K. Reduced silicone oil adherence to silicone intraocular lens by surface modification with 2-methacryloyloxyethyl phosphoryl-choline. Curr Eye Res. 2013;38(1):91-96.

12. Yao K, Huang XD, Huang XJ, Xu ZK. Improvement of the surface biocompatibility of silicone intraocular lens by the plasma-induced tethering of phospholipid moieties. J Biomed Mater Res A. 2006;78(4): 684-692.

13. Ishikawa N, Miyamoto T, Okada Y, Saika S. Cell adhesion on explanted intraocular lenses part 2: experimental study of a surface-modified IOL in rabbits. J Cataract Refract Surg. 2011;37(7):1339-1342.

14. D'Sa RA, Burke GA, Meenan BJ. Lens epithelial cell response to atmospheric pressure plasma modified poly(methylmethacrylate) surfaces. J Mater Sci Mater Med. 2010;21(5):1703-1712. 
15. D'Sa RA, Dickinson PJ, Raj J, Pierscionek BK, Meenan BJ. Inhibition of lens epithelial cell growth via immobilisation of hyaluronic acid on atmospheric pressure plasma modified polystyrene. Soft Matter. 2011;7(2): 608-617.

16. Hata Viveiros MM, Soares RT, Model MS, et al. Adhesion study of cultured human lens capsule cells on hydrophilic intraocular lenses coated with polyethylene glycol. J Cataract Refract Surg. 2015;41(7): 1478-1483.

17. Keefe AJ, Brault ND, Jiang S. Suppressing surface reconstruction of superhydrophobic PDMS using a superhydrophilic zwitterionic polymer. Biomacromolecules. 2012;13(5):1683-1687.

18. Audouin F, Heise A. Surface-initiated RAFT polymerization of NIPAM from monolithic macroporous polyHIPE. Eur Polym J. 2013;49(5): 1073-1079.

19. Yuan J, Huang X, Li P, Li L, Shen J. Surface-initiated RAFT polymerization of sulfobetaine from cellulose membranes to improve hemocompatibility and antibiofouling property. Polym Chem. 2013;4(19):5074-5085.

20. Chen X, Shang H, Cao S, Tan H, Li J. A zwitterionic surface with general cell-adhesive and protein-resistant properties. RSC Adv. 2015;5(93): 76216-76220.

21. Lin QK, Xu X, Wang BL, et al. Hydrated polysaccharide multilayer as an intraocular lens surface coating for biocompatibility improvements. J Mater Chem B. 2015;3(18):3695-3703.

22. Wang BL, Jin TW, Han YM, et al. Surface-initiated RAFT polymerization of $\mathrm{p}$ (MA POSS-co-DMAEMA+) brushes on PDMS for improving anti-adhesive and antibacterial properties. Int J Polym Mater. 2016;65(2):55-64

23. Özçam AE, Roskov KE, Spontak RJ, Genzer J. Generation of functional PET microfibers through surface-initiated polymerization. J Mater Chem. 2012;22(12):5855-5864.

24. Ji Y, Wei Y, Liu XS, Wang JL, Ren KF, Ji J. Zwitterionic polycarboxybetaine coating functionalized with REDV peptide to improve selectivity for endothelial cells. J Biomed Mater Res A. 2012;100(6):1387-1397.

25. Chen YJ, Liu XS, Wang HB, Wang Y, Jin Q, Ji J. Zwitterions in surface engineering of biomedical nanoparticles. Prog Chem. 2014;26(11): 1849-1858.

26. Yeh SB, Chen CS, Chen WY, Huang CJ. Modification of silicone elastomer with zwitterionic silane for durable antifouling properties. Langmuir. 2014;30(38):11386-11393.

27. Huang CJ, Chu SH, Wang LC, Li CH, Lee TR. Bio-inspired zwitterionic surface coatings with robust photostability and fouling resistance. ACS Appl Mater Interfaces. 2015;7(42):23776-23786.

28. Werner L. Biocompatibility of intraocular lens materials. Curr Opin Ophthalmol. 2008;19(1):41-49.

29. Bozukova D, Pagnoulle C, Pauw-Gillet MCD, et al. Improved performances of intraocular lenses by poly(ethylene glycol) chemical coatings. Biomacromolecules. 2007;8(8):2379-2387.

30. McCoy CP, Craig RA, McGlinchey SM, Carson L, Jones DS, Gorman SP. Surface localisation of photosensitisers on intraocular lens biomaterials for prevention of infectious endophthalmitis and retinal protection. Biomaterials. 2012;33(32):7952-7958.
31. Klintworth GK. Cornea - structure and macromolecules in health and disease. Am J Pathol. 1977;89(3):718-808.

32. Awasthi N, Wang-Su ST, Wagner BJ. Downregulation of MMP-2 and -9 by proteasome inhibition: a possible mechanism to decrease LEC migration and prevent posterior capsular opacification. Invest Ophthalmol Vis Sci. 2008;49(5):1998-2003.

33. Argal S. Newer intraocular lens materials and design. J Clin Ophthalmol Res. 2013;1(2):113-117.

34. Buehl W. Effect of intraocular lens design on posterior capsule opacification. J Cataract Refract Surg. 2008;34(11):1976-1985.

35. Pei C, Xu Y, Jiang JX, Cui LJ, Li L, Qin L. Application of sustained delivery microsphere of cyclosporine A for preventing posterior capsular opacification in rabbits. Int J Ophthalmol. 2013;6(1):1-7.

36. Wang BL, Lin QK, Shen CH, Han YM, Tang JM, Chen H. Synthesis of MA POSS-PMMA as an intraocular lens material with high light transmittance and good cytocompatibility. RSC Adv. 2014;4(95): 52959-52966.

37. Wang BL, Lin QK, Shen CH, Tang JM, Han YM, Chen H. Hydrophobic modification of polymethyl methacrylate as intraocular lenses material to improve the cytocompatibility. J Colloid Interface Sci. 2014;431:1-7.

38. Shen C, Han Y, Wang B, Tang J, Chen H, Lin Q. Ocular biocompatibility evaluation of POSS nanomaterials for biomedical material applications. RSC Adv. 2015;5(66):53782-53788.

39. Lin QK, Yan JJ, Ji J, Shen JC. Natural anticoagulant and endothelial cell-compatible multilayer for endovascular stents surface modification. Chem J Chin Univ. 2009;30(6):1256-1258.

40. Lin QK, Hou Y, Xu X, et al. Anti-CD34 antibody functionalized swollen polymeric coating for endothelial cell rapid selectively capture. Int J Polym Mater. 2015;64(2):99-103.

41. Wei Y, Ji Y, Xiao LL, et al. Surface engineering of cardiovascular stent with endothelial cell selectivity for in vivo re-endothelialisation. Biomaterials. 2013;34(11):2588-2599.

42. Lin QK, Hou Y, Ren KF, Ji J. Selective endothelial cells adhesion to Arg-Glu-Asp-Val peptide functionalized polysaccharide multilayer. Thin Solid Films. 2012;520(15):4971-4978.

43. Tang JM, Han YM, Chen H, Lin QK. Bottom-up fabrication of PEG brush on poly(dimethylsiloxane) for anti-fouling surface construction. Int J Polym Sci. 2016;2016:8458752.

44. Xu X, Tang JM, Han YM, Wang W, Chen H, Lin QK. Surface PEGylation of intraocular lens for PCO prevention: an in vivo evaluation. J Biomater Appl. 2016;31(1):68-76.

45. Lin QK, Xu X, Wang YQ, Wang BL, Chen H. Antiadhesive and antibacterial polysaccharide multilayer as IOL coating for prevention of postoperative infectious endophthalmitis. Int J Polym Mater. In press 2016.
International Journal of Nanomedicine

\section{Publish your work in this journal}

The International Journal of Nanomedicine is an international, peerreviewed journal focusing on the application of nanotechnology in diagnostics, therapeutics, and drug delivery systems throughout the biomedical field. This journal is indexed on PubMed Central, MedLine, CAS, SciSearch $®$, Current Contents $\AA /$ Clinical Medicine,

\section{Dovepress}

Journal Citation Reports/Science Edition, EMBase, Scopus and the Elsevier Bibliographic databases. The manuscript management system is completely online and includes a very quick and fair peer-review system, which is all easy to use. Visit http://www.dovepress.com/ testimonials.php to read real quotes from published authors. 